ESJ Humanities

\title{
Genre et pouvoir de prise de décision du type et de l'heure de repas: implications pour la sécurité alimentaire des ménages au Nord-Bénin (Afrique de l'Ouest)
}

\author{
Janvier Egah \\ Laboratoire Société- Environnement (LaSEn), Faculté d'Agronomie, \\ Université de Parakou, Bénin \\ Université Catholique de Louvain, Belgique \\ Ibrahim El Ghazi \\ Université Moulay Ismail, Cluster des Compétences \\ Environnement et santé, Meknès, Maroc \\ Université Catholique de Louvain, Belgique \\ Mohamed Nasser Baco \\ Laboratoire Société- Environnement (LaSEn), Faculté d'Agronomie, \\ Université de Parakou, Bénin \\ Marie-Paule Kestemont \\ Université Catholique de Louvain, Belgique
}

Doi:10.19044/esj.2021.v17n33p241

Submitted: 03 August 2021

Accepted: 23 August 2021

Published: 30 September 2021
Copyright 2021 Author(s)

Under Creative Commons BY-NC-ND

4.0 OPEN ACCESS

Cite As:

Egah J., El Ghazi I., Baco M.N. \& Kestemont M-P. (2021). Genre et pouvoir de prise de décision du type et de l'heure de repas: implications pour la sécurité alimentaire des ménages au Nord-Bénin (Afrique de l'Ouest). European Scientific Journal, ESJ, 17 (33), 241. https://doi.org/10.19044/esj.2021.v17n33p241

\section{Résumé}

Le genre se trouve au centre des débats sur l'insécurité alimentaire des ménages. La présente étude vise à analyser l'influence de la participation des deux sexes au processus de prise de décision de l'heure de prise de repas et du type de repas consommé dans les ménages. Elle a été réalisée au Nord-Bénin auprès de 295 ménages aléatoirement sélectionnés. Les données collectées à l'aide d'un questionnaire digitalisé ont porté sur les caractéristiques socioéconomiques, le sexe du preneur de décision de l'heure de prise de repas et du type de repas consommé, les huit questions de l'échelle d'expérience de l'insécurité alimentaire des ménages. La statistique descriptive et la régression linéaire ont été utilisées pour analyser les données. Les résultats révèlent que 
la prise de décision unilatérale de l'heure de prise de repas par l'homme ou la femme a affecté négativement le niveau de sécurité alimentaire des ménages par rapport aux ménages dans lesquels la décision est prise par les deux sexes. Le choix du type de repas par l'homme a amélioré le niveau de sécurité alimentaire des ménages plus que les ménages où la décision est bilatérale. La dépense alimentaire de l'homme a négativement affecté le niveau de sécurité alimentaire des ménages alors que celle de la femme a contribué à son amélioration. La taille de ménage a influencé positivement le niveau de sécurité alimentaire des ménages. La promotion du genre doit être renforcée pour faciliter les décisions bilatérales de l'heure de prise de repas et responsabiliser les hommes face aux dépenses alimentaires.

Mots-clés: Genre, sécurité alimentaire, dépense alimentaire, prise de décision, Afrique de l'Ouest

\title{
Gender and Decision-Making Power of Meal Type and Time: Implications for Household Food Security in North Benin (West Africa)
}

\author{
Janvier Egah \\ Laboratoire Société- Environnement (LaSEn), Faculté d'Agronomie, \\ Université de Parakou, Bénin \\ Université Catholique de Louvain, Belgique \\ Ibrahim El Ghazi \\ Université Moulay Ismail, Cluster des Compétences \\ Environnement et santé, Meknès, Maroc \\ Université Catholique de Louvain, Belgique \\ Mohamed Nasser Baco \\ Laboratoire Société- Environnement (LaSEn), Faculté d'Agronomie, \\ Université de Parakou, Bénin \\ Marie-Paule Kestemont \\ Université Catholique de Louvain, Belgique
}

\begin{abstract}
Gender is at the center of the debate on household food insecurity. This study aims to analyze the influence of gender participation in the decisionmaking process of mealtime and type of meal consumed in households. A sample of 295 households was randomly selected to be surveyed in NorthBenin. Data collected using a digitized questionnaire included socioeconomic characteristics, gender of the decision-maker of mealtime and type of meal
\end{abstract}


consumed, the eight questions of the Household Food Insecurity Experience Scale, etc. Data were analyzed using descriptive statistics and linear regression. The results reveal that unilateral decision-making on mealtimes by either the man or the woman negatively affected the level of household food security more than households in which the decision is made by both sexes. Man's choice of meal type improved household food security more than households where the decision is bilateral. Man's food expenditure negatively affected the level of household food security, while women's expenditure has contributed to its improvement. Household size positively influenced the level of household food security. Gender promotion should be strengthened to facilitate bilateral decisions of mealtime and empower men to take responsibility for the food expenditures of their households.

Keywords: Gender, food security, food expenditure, decision-making, West Africa

\section{Introduction}

L'insécurité alimentaire constitue un phénomène qui sévit dans le monde entier. La sécurité alimentaire d'un ménage est assurée lorsque ce dernier a matériellement, socialement et économiquement accès de façon permanente, à une nourriture suffisante, saine et nutritive pour satisfaire ses besoins et préférences alimentaires afin de mener une vie saine et active (FAO et al., 2020). Ces organisations ont déclaré qu'environ 8,9\% de la population est sous-alimentée dans le monde. Cette prévalence de l'insécurité alimentaire est élevée en Afrique subsaharienne où environ $22 \%$ de la population souffre des formes graves de la sous-alimentation et de l'insécurité alimentaire (FAO et al., 2020). Le Bénin, l'un des pays de l'Afrique de l'Ouest, constitue un épicentre de l'insécurité alimentaire. En effet, selon l'Analyse Globale de la Vulnérabilité et de la Sécurité Alimentaire, environ 1,09 millions de personnes, soit 9,6\% des ménages, vivent en insécurité alimentaire au Bénin (Institut National de la Statistique et de 1'Analyse Economique (INSAE) et Programme Alimentaire Mondial (PAM), 2017). Aussi, 42,9\% des ménages vivent-ils dans des conditions de sécurité alimentaire limite. En effet, l'économie du Bénin est fortement liée à l'agriculture qui emploie plus de 50\% de la population active (Bayé, 2018). Selon INSAE (2016), environ 55\% de la population vit en milieu rural et $50 \%$ des femmes vivent dans les ménages agricoles. La contribution de l'agriculture au Produit Intérieur Brut (PIB) est près de $20 \%$. Près de $75 \%$ des recettes d'exportation viennent de l'agriculture (Bayé, 2018).

Face à l'insécurité alimentaire des populations, surtout rurales, le Gouvernement du Bénin, à l'instar des communautés internationales, a initié des actions de lutte contre la faim et l'insécurité alimentaire dans toutes ses 
formes. Ces actions s'inscrivent des documents stratégiques tels que le Plan Stratégique de Développement du Secteur Agricole (PSDSA) et le Plan d'actions d'Alimentation et de Nutrition dans le secteur agricole à l'horizon 2016-2020. Ces documents visent à opérationnaliser le Plan Stratégique de Développement de l'Alimentation et de la Nutrition (PSDAN) pour contribuer aux piliers de la disponibilité, de l'accessibilité et de l'utilisation alimentaire. Dans ces documents politiques, un accent particulier a été mis sur l'importance de la prise en compte du genre dans la mise en œuvre des politiques de développement. De plus, depuis 2017, selon la FAO et la Commission de la CEDEAO (2018), des actions et mesures pour l'amélioration de l'accès des femmes aux ressources (terre, intrants agricoles, accompagnement pour la valorisation des productions agricoles et emplois rémunérateurs), le renforcement des capacités d'actions des femmes rurales et le développement d'un environnement institutionnel et juridique favorable ont été prévues dans le Plan National d'Investissement Agricole, de la Sécurité Alimentaire et Nutritionnelle (PNIASAN) 2017-2021. En juillet 2021, le Gouvernement a adopté, en Conseil des Ministres du mercredi 21 juillet 2021, la création de l'Institut National de la Femme au Bénin pour l'épanouissement des femmes et leur participation à la prise de décision. Ces différents engagements du Gouvernement du Bénin doivent être accompagnés par la recherche pour résorber le problème d'insécurité alimentaire.

À cet égard, plusieurs études ont montré que le revenu, la production agricole, etc. influencent la sécurité alimentaire (Dury et al., 2017). La gestion des ressources à l'intérieur des ménages en faveur des femmes constitue un important facteur pour lutter contre l'insécurité alimentaire des ménages en Haïti (Pauzé, 2015). Felker-Kantor et Wood (2012) ont montré que les femmes investissent plus que les hommes dans les besoins sociaux, y compris l'alimentation des membres de leurs ménages. Lourme-Ruiz et al. (2016) ont révélé que l'accès des femmes au revenu du ménage garantit une meilleure diversité alimentaire de leurs ménages. Malgré ces rôles cruciaux de la femme dans la lutte contre l'insécurité alimentaire, les femmes continuent de subir une injustice sociale ou une discrimination qui ne leur permet pas de participer au processus de décision en matière d'alimentation dans leurs ménages (Verschuur, 2011). Elles ne jouissent pas de la parité homme femme pour participer efficacement à la lutte contre l'insécurité alimentaire de tous les membres de leurs ménages (Oussou et al., 2017). Aboubakar (2017) notifie que les femmes continuent de subir des discriminations et heurtent des barrières socioculturelles telles que le poids des préjugés, les perceptions culturelles concernant le rôle des femmes, etc.

À ce propos, il s'avère indispensable de questionner la participation des femmes au processus de prise de décision dans les ménages, notamment en ce qui concerne le choix des types de repas à consommer et l'heure de prise 
de repas dans les ménages. C'est donc l'objectif de cette étude qui vise à analyser les effets de la participation des femmes au processus de prise de décision relative à l'alimentation et aux dépenses alimentaires dans les ménages ruraux du Bénin.

\section{Matériels et méthode}

\subsection{Présentation de la zone d'étude}

L'étude a été menée spécifiquement au Nord-Bénin dans les Communes de Sinendé et de Bembèrèké, fortement agricoles dans le département du Borgou (Figure 1). Ces communes appartiennent au pôle de développement agricole $\mathrm{N}^{\circ} 2$. Au niveau de ce pôle, la culture de coton est la culture locomotive alors que les filières de diversification sont le maïs, le sorgho, le soja, les bovins, les petits ruminants et les volailles (Bureau d'études et d'Appui au secteur Agricole (B2A), 2016).

La Commune de Sinendé est limitée au sud par la Commune de N'Dali, au nord par la Commune de Gogounou, à l'ouest par les Communes de Ouassa-Péhunco et de Djougou et à l'Est par la Commune de Bembèrèkè. Elle occupe $8,85 \%$ de la superficie du département du Borgou, avec une superficie de $2289 \mathrm{~km}^{2}$. Elle est caractérisée par un relief de plateaux et de collines sur le prolongement ouest de mont Bembéréké. La Commune de Sinendé est arrosée par un climat de type soudano- guinéen qui lui confère une saison pluvieuse de six mois (mai à octobre) et une saison sèche (novembre à avril). La hauteur d'eau annuellement enregistrée varie entre $1000 \mathrm{~mm}$ et $1200 \mathrm{~mm}$ avec le maximum de précipitations aux mois d'août et de septembre. La population de Sinendé est de 91672 habitants dont 61,5\% résident en zone rurale et 50,2\% sont des femmes. Quant à la Commune de Bembéréké, plus vaste que Sinendé, elle couvre $12,94 \%$ de la superficie du département du Borgou avec une superficie de $3348 \mathrm{~km}^{2}$. Elle est limitée au sud par la Commune de N'Dali, au nord par la Commune de Gogounou, à l'ouest par celle de Sinendé et à l'est par les Communes de Kalalé et de Nikki. Elle est marquée par une vaste pénéplaine granito-gnésique avec une rupture sporadique de l'existence de chaînons de collines communément appelées «Monts de BEMBEREKE ». La Commune est arrosée par un climat de type soudano-guinéen avec une grande saison de pluies (avril à octobre) et une grande saison sèche (novembre- mars). La pluviométrie moyenne annuelle, comme à Sinendé, se situe entre 1000 et $1200 \mathrm{~mm}$. L'effectif de la population de Bembèrèké est de 131255 habitants dont 59\% vivent en milieu rural et $49,5 \%$ sont de femmes (INSAE, 2016). 

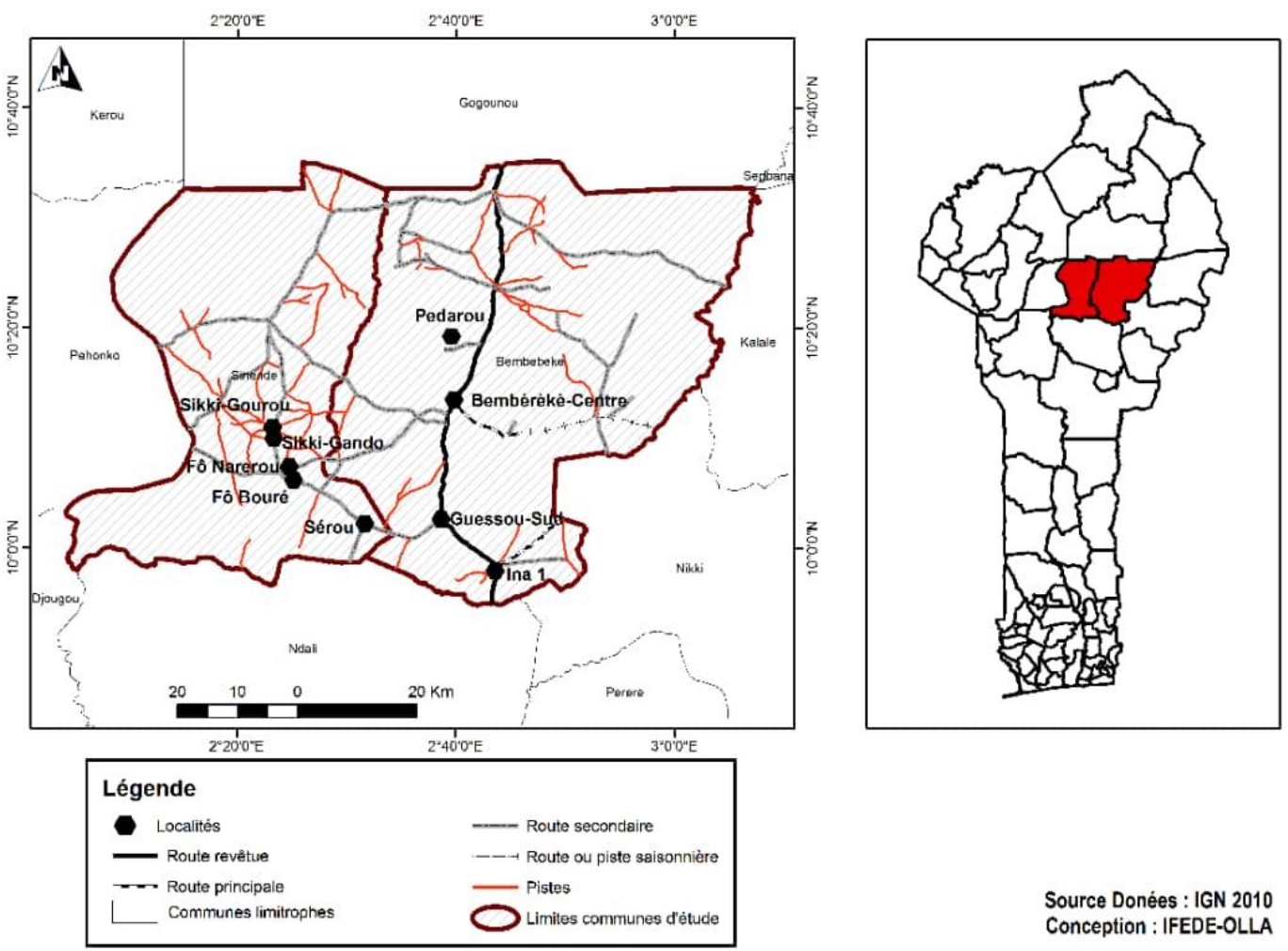

Figure 1 : Carte de la zone d'étude

Source Donées : IGN 2010 Conception : IFEDE-OLLA

\subsection{Unités de recherche et échantillonnage}

L'étude s'est inspirée de l'approche quantitative en socioéconomie de développement. Ainsi, une démarche quantitative a été utilisée pour l'échantillonnage et la collecte des données. L'échantillonnage aléatoire simple a été effectué choisir les villages et les ménages (Tableau 1). Les ménages ont été les unités d'investigation de cette étude. Dans chaque ménage, les entretiens ont eu lieu avec l'homme et la femme (ou les femmes) du ménage. Au total, 295 ménages ont été enquêtés de façon aléatoire simple. La différence de la taille de l'échantillon entre les deux Communes est due à la non validité des données collectées auprès de 5 ménages de la Commune de Bembèrèké.

Tableau 1 : Villages d'étude et taille d'échantillon

\begin{tabular}{cll}
\hline Commune & Villages & Total des enquêtés \\
\hline \multirow{5}{*}{ Bembèrè̀è } & Bembèrèké centre & 9 \\
\cline { 2 - 3 } & Guessou-Sud & 50 \\
\cline { 2 - 3 } & Ina 1 & 45 \\
\cline { 2 - 3 } & Pedarou & 41 \\
\cline { 2 - 3 } Sidendé & Sous-total & $\mathbf{1 4 5}$ \\
\hline & Fô Bouré & 43 \\
\cline { 2 - 3 } & Fô Narerou & 7 \\
\hline
\end{tabular}




\begin{tabular}{lll}
\hline & Sérou & 50 \\
\cline { 2 - 3 } & Sikki Gando & 25 \\
\cline { 2 - 3 } & Sikki Gourou & 25 \\
\cline { 2 - 3 } & Sous-total & $\mathbf{1 5 0}$ \\
\hline Zone d'étude & & $\mathbf{2 9 5}$ \\
\hline
\end{tabular}

Source : Enquête de terrain, Mars 2020.

\subsection{Collecte de données}

Les données collectées ont porté sur les caractéristiques socioéconomiques des ménages enquêtés telles que l'âge du chef de ménage, la taille du ménage, le sexe du décideur de type de repas consommé, le sexe du décideur de l'heure de prise de repas, les dépenses alimentaires annuelles effectuées par l'homme et par la femme au profit des membres des ménages, les huit questions standards de l'échelle d'expérience de l'insécurité alimentaire des ménages (HFIES) mise au point par la FAO (2016) pour mesurer le niveau de sécurité alimentaire des ménages (Tableau 2).

Les données ont été collectées à l'aide d'un questionnaire lors des entretiens individuels avec l'homme et la femme (ou les femmes) dans les ménages.

\subsection{Analyse de données}

L'analyse des données a été faite à l'aide de la statistique descriptive et de la régression linéaire. L'HFIES a été calculée en faisant la somme des réponses affirmatives. La prévalence des ménages à l'insécurité alimentaire a été appréciée selon les classes de niveau de sécurité alimentaire (Tableau 2).

Tableau 2 : Classification de l'HFIES

\begin{tabular}{|c|c|}
\hline Niveau de sécurité alimentaire & Classe de HFIES \\
\hline Sécurité alimentaire & 0 \\
\hline Insécurité alimentaire légère & $1-3$ \\
\hline Insécurité alimentaire modérée & $4-6$ \\
\hline Insécurité alimentaire sévère & $7-8$ \\
\hline
\end{tabular}

La statistique descriptive a permis de calculer les paramètres tels que la moyenne et l'écart-type des variables quantitatives (âge du chef de ménage, taille du ménage, dépense alimentaire effectuée par chaque sexe) et les fréquences des décideurs du type de repas et de l'heure de prise de repas selon le sexe. Quant à la régression linéaire, le test de corrélation a permis d'identifier les variables explicatives autocorrélées pour lever les cas de multicolinéarité. Pour des raisons de parcimonie, les variables n'ayant pas de coefficients significatifs ont été exclues du modèle et le modèle a été repris. Ainsi, l'âge et le niveau d'éducation du chef de ménage ont été exclus du modèle. Les variables retenues dans le modèle sont présentées dans le tableau 3. L'HFIES, étant un indicateur de mesure d'insécurité alimentaire des 
ménages variant de 0 à 8 , il a été inversé pour faciliter les interprétations des résultats. Ainsi, plus l'HFIES est élevé, plus le ménage est en sécurité alimentaire.

Tableau 3 : Spécification du modèle de régression linéaire

\begin{tabular}{|c|c|c|c|c|}
\hline Variables & Nature de variable & Modalités & $\begin{array}{l}\text { Signe } \\
\text { prévu }\end{array}$ & Source \\
\hline \multicolumn{5}{|c|}{$\begin{array}{ll}\text { Variable dépendante } \\
\end{array}$} \\
\hline $\begin{array}{lcc}\text { Niveau de } & \text { sécurité } \\
\text { alimentaire } & \text { HFIES } & \end{array}$ & $\begin{array}{c}\text { Variable } \\
\text { discontinue }\end{array}$ & & & \\
\hline \multicolumn{5}{|c|}{$\begin{array}{ll}\text { Variables indépendantes } \\
\end{array}$} \\
\hline $\begin{array}{c}\text { Définition de l'heure de } \\
\text { prise de repas par l'homme }\end{array}$ & Variable nominale & $\begin{array}{l}1=\text { oui } ; 0= \\
\text { non }\end{array}$ & $+/-$ & $\begin{array}{l}\text { Barou et Verhoeven, } \\
1997\end{array}$ \\
\hline $\begin{array}{l}\text { Définition de l'heure de } \\
\text { prise de repas par la femme }\end{array}$ & Variable nominale & $\begin{array}{l}1=\text { oui } ; 0= \\
\text { non }\end{array}$ & + & $\begin{array}{l}\text { Le Pape et Plessz, } \\
2017 \text {; Mohamed- } \\
\text { Katerere et Smith, } \\
2013\end{array}$ \\
\hline $\begin{array}{l}\text { Choix du type de repas par } \\
\text { l'homme }\end{array}$ & Variable nominale & $\begin{array}{l}1=\text { oui } ; 0= \\
\text { non }\end{array}$ & $+/-$ & $\begin{array}{l}\text { Barou et Verhoeven, } \\
1997\end{array}$ \\
\hline $\begin{array}{l}\text { Choix du type de repas par } \\
\text { la femme }\end{array}$ & Variable nominale & $\begin{array}{l}1=\text { oui } ; 0= \\
\text { non }\end{array}$ & + & $\begin{array}{l}\text { Le Pape et Plessz, } \\
2017 \text {; Mohamed- } \\
\text { Katerere et Smith, } \\
2013\end{array}$ \\
\hline $\begin{array}{c}\text { Dépense alimentaire de } \\
\text { l'homme }\end{array}$ & Variable continue & & + & $\begin{array}{l}\text { Barou et Verhoeven, } \\
1997\end{array}$ \\
\hline $\begin{array}{l}\text { Dépense alimentaire de la } \\
\text { femme }\end{array}$ & Variable continue & & + & $\begin{array}{l}\text { Le Pape et Plessz, } \\
2017 \text {; Mohamed- } \\
\text { Katerere et Smith, } \\
2013\end{array}$ \\
\hline Taille du ménage & Variable continue & & - & Farzaneh et al., 2017 \\
\hline
\end{tabular}

\section{Résultats et discussions}

\subsection{Caractéristique des ménages}

Les chefs de ménages enquêtés ont un âge moyen de 37 ans (+/-10,57 ans). Ils ont un niveau moyen d'éducation primaire. La taille du ménage est de 4 personnes en moyenne. La dépense alimentaire effectuée par l'homme dans le ménage est en moyenne de $217008 \mathrm{FCFA} /$ an alors que celle de la femme est de 103015 FCFA/ an. Ainsi, les femmes ont moins dépensé que les hommes pour l'alimentation des membres de leurs ménages. Ces résultats sont contraires aux assertions de Felker-Kantor et Wood (2012) qui ont montré que les femmes contribuent plus aux dépenses alimentaires que les hommes. Cette différence pourrait s'expliquer par le niveau de revenu des femmes comparativement à celui des hommes. 
Tableau 4: Caractéristiques des ménages

\begin{tabular}{|c|c|c|c|c|c|}
\hline & $\mathrm{N}$ & Minimum & Maximum & Moyenne & Ecart type \\
\hline Age & 295 & 11,0 & 80,0 & 37,01 & 10,57 \\
\hline Niveau d'éducation & 295 & 0,0 & 3,0 & 0,66 & 0,90 \\
\hline Taille du ménage & 295 & 0,0 & 18,0 & 3,97 & 3,20 \\
\hline Dépense alimentaire de l'homme & 295 & 0,0 & 1224000,0 & 217008,33 & 178658,96 \\
\hline Dépense alimentaire de la femme & 295 & 0,0 & 624000,0 & 103014,59 & 153282,03 \\
\hline
\end{tabular}

\subsection{Prévalence de l'insécurité alimentaire}

Les ménages enquêtés ont un niveau moyen de sécurité alimentaire de 4,8 (+/- 3.2). L'étude a révélé qu'environ 34\% des ménages enquêtés sont en sécurité alimentaire. Par contre, environ $26 \%$ des ménages enquêtés sont en insécurité alimentaire sévère (Tableau 4). Ce résultat montre que la prévalence de l'insécurité alimentaire dans la zone d'étude est supérieure à celle obtenue au niveau national en 2017 (INSAE et PAM, 2017). En effet, l'analyse globale de la vulnérabilité et de la sécurité alimentaire a révélé qu'environ 9,6\% des ménages sont en insécurité alimentaire et $42,9 \%$ des ménages vivent dans des conditions de sécurité alimentaire limite (INSAE et PAM, 2017).

Tableau 4: Prévalence de l'insécurité alimentaire

\begin{tabular}{|c|c|c|}
\hline Niveau de sécurité alimentaire & Effectif & Fréquence (\%) \\
\hline Sécurité alimentaire & 100 & 33,9 \\
\hline Insécurité alimentaire légère & 78 & 26,4 \\
\hline Insécurité alimentaire modérée & 39 & 13,2 \\
\hline Insécurité alimentaire sévère & 78 & 26,4 \\
\hline Total & 295 & 100,0 \\
\hline
\end{tabular}

\subsection{Décideurs du type de repas et de l'heure de prise de repas}

Dans la majorité des ménages enquêtés (55\%), le choix du type de repas à consommer par les membres du ménage est fait bilatéralement par l'homme et la femme. Par contre, l'homme a décidé du type de repas consommé dans $17 \%$ environ des ménages enquêtés (Tableau 5). Ainsi, on peut dire que la décision du type de repas consommé par les membres du ménage est faite par l'homme et la femme.

En ce qui concerne l'heure de consommation du repas, elle est décidée bilatéralement par l'homme et la femme dans la majorité des ménages enquêtés (70\%). Toutefois, l'homme a décidé de l'heure de prise de repas dans $8 \%$ des ménages. La femme a décidé de l'heure de prise de repas dans environ $22 \%$ des ménages enquêtés. 
Tableau 5 : Décideur du type de repas consommé et de l'heure de prise de repas

\begin{tabular}{|c|c|c|c|}
\hline & Modalités & Effectif & Fréquence (\%) \\
\hline \multirow{2}{*}{$\begin{array}{c}\text { Décideur du type } \\
\text { de repas }\end{array}$} & Homme & 50 & 16,9 \\
\cline { 2 - 4 } & Femme & 83 & 28,1 \\
\cline { 2 - 4 } & Les deux & 161 & 54,6 \\
\hline \multirow{2}{*}{$\begin{array}{c}\text { Décideur de } \\
\text { l'heure de repas }\end{array}$} & Homme & 23 & 7,8 \\
\cline { 2 - 4 } & Femme & 64 & 21,7 \\
\cline { 2 - 4 } & Les deux & 207 & 70,2 \\
\hline
\end{tabular}

\subsection{Influence du sexe du décideur sur la sécurité alimentaire des ménages}

La régression linéaire effectuée est globalement significative au seuil de $1 \%$. Le pouvoir explicatif $\mathrm{R}^{2}$ du modèle est de $24 \%$ (Tableau 6). Ainsi, $24 \%$ de la variation de HFIES sont expliquées par l'ensemble des variables explicatives introduites dans le modèle.

Tableau 6 : Influence du sexe de décideur et des dépenses alimentaires sur la sécurité alimentaire des ménages

\begin{tabular}{|c|c|c|c|c|}
\hline Variables explicatives & Coefficients & $\begin{array}{c}\text { Erreur } \\
\text { standard }\end{array}$ & T & Sig. \\
\hline Constante & 4,469 & 0,362 & 12,352 & 0,000 \\
\hline $\begin{array}{c}\text { Décision de l'heure de prise de repas } \\
\text { par l'homme }\end{array}$ & $-3,805$ & 0,709 & $-5,369$ & 0,000 \\
\hline $\begin{array}{c}\text { Décision de l'heure de prise de repas } \\
\text { par la femme }\end{array}$ & $-1,923$ & 0,581 & $-3,311$ & 0,001 \\
\hline Choix du type de repas par l'homme & 1,277 & 0,528 & 2,420 & 0,016 \\
\hline Choix du type de repas par la femme & 0,604 & 0,530 & 1,140 & 0,255 \\
\hline Dépense alimentaire de l'homme & $-2,170 \mathrm{E}-06$ & 0,000 & $-2,251$ & 0,025 \\
\hline Dépense alimentaire de la femme & $2,480 \mathrm{E}-06$ & 0,000 & 1,956 & 0,051 \\
\hline Taille du ménage & 0,227 & 0,060 & 3,772 & 0,000 \\
\hline Test de régression & \multicolumn{3}{|c|}{$\mathrm{R}^{2}=24 \% ; \mathrm{ddl}=7 ; \mathrm{p}=0,000$} \\
\hline
\end{tabular}

De l'analyse du tableau 6, il ressort que la décision de l'heure de prise de repas par l'homme ou par la femme a négativement influencé le niveau de sécurité alimentaire des ménages par rapport à la décision bilatérale de l'heure de prise de repas au seuil de $1 \%$ car les coefficients de ces variables sont négatifs et significatifs au seuil de $1 \%$. En effet, il a été prouvé par plusieurs études que les heures de prise de repas sont aux mains des femmes (MohamedKaterere et Smith, 2013 ; Le Pape et Plessz, 2017). Ces auteurs ont montré que les femmes consacrent collectivement près de 8 milliards d'heures de travail y compris la cuisine. Le Pape et Plessz (2017) ont montré que les femmes influencent surtout l'heure de prise de repas du matin et s'attachent à la qualité des repas que leurs enfants prennent avant d'aller à l'école. Mais cette étude vient de montrer que le choix bilatéral des heures de prise de repas est plus bénéfique en termes de sécurité alimentaire des ménages que le choix unilatéral par un sexe. 
Quant au choix du type de repas consommé, lorsqu'il est effectué par l'homme, le coefficient est positif et significatif au seuil de 5\%. Par contre, le choix du type de repas par la femme n'a pas influencé le niveau de sécurité alimentaire des ménages car son coefficient n'est pas significatif au seuil de $5 \%$. Ainsi, le choix de l'homme en ce qui concerne le type de repas consommé a positivement influencé le niveau de sécurité alimentaire du ménage comparativement à un choix bilatéral des deux sexes. Ce résultat suggère que lorsque l'homme décide du type de repas consommé dans son ménage, il met les moyens pour garantir une alimentation équilibrée et diversifiée. Ce résultat contrarie celui de Le Pape et Plessz (2019), qui ont montré que les femmes assurent un contrôle rapproché ou à distance de la qualité des repas consommés dans les ménages. La différence peut s'expliquer par le fait que ces femmes prennent en charge le petit-déjeuner des enfants ; ce qui n'est pas le cas dans cette étude.

En ce qui concerne la dépense alimentaire, la dépense alimentaire effectuée par l'homme au profit du ménage a un coefficient négatif et significatif au seuil de 5\%, alors que celle de la femme a un coefficient positif et significatif au seuil de $10 \%$. Ce constat pourrait être expliqué par le fait que les hommes achètent plus les aliments de base tels que le maïs, le sorgho et l'igname. Par contre, les femmes, lorsqu'elles ont l'accès aux revenus dans les ménages, elles achètent des aliments complémentaires pour diversifier les repas (Lourme-Ruiz et al., 2016). Ces auteurs ont montré dans une étude que les femmes développent des stratégies culinaires pour capter l'attention de leurs maris. L'une de ces stratégies consistent à acheter les légumes (gombo, épinard, épices, etc.) pour agrémenter les repas de leurs maris et de leurs enfants (Barou et Verhoeven, 1997). Les femmes consacrent plus leur revenu aux compléments alimentaires du ménage, notamment les légumes, les espèces halieutiques, etc. qui sont très essentielles pour l'alimentation (Barou et Verhoeven, 1997; Weiant et Aswani, 2006). Ainsi, elles contribuent à une meilleure diversité alimentaire de leurs ménages. Par contre, les hommes ravitaillent leurs ménages en aliments de base (manioc, mil, etc.) qui ne permettent pas aux ménages de diversifier leurs alimentations (Barou et Verhoeven, 1997).

Dans cette étude, la taille du ménage a influencé positivement le niveau de sécurité alimentaire car son coefficient est positif et significatif au seuil de $1 \%$. Ce résultat sous-entend que dans des ménages de grande taille, il existe plusieurs actifs agricoles qui contribuent à la production agricole vivrière. Ces actifs agricoles assurent, la disponibilité alimentaire dans les ménages. Ainsi, ils contribuent à la diversification des sources de nourriture, favorisant ainsi l'accès à des aliments sains, équilibrés et variés. Ce résultat rejoint l'assertion de Weiant et Aswani (2006) selon lesquels les ménages ayant une taille importante jouissent des emplois réguliers et ont une meilleure diversification 
des aliments. Toutefois, le résultat contredit celui rapporté par une étude menée en Iran et qui a montré que l'insécurité alimentaire avait une corrélation significativement positive avec le nombre de membres du ménage (Farzaneh et al., 2017). Cette différence pourrait se justifier par le fait que l'indicateur utilisé par ces auteurs est la fréquence alimentaire basée sur 18 questions alors que cette étude s'est axée sur l'HFIES de la FAO (2016).

\section{Conclusion}

L'étude a montré l'importance de la participation des deux sexes au processus de prise de décision des types de repas et des heures de prise de repas dans les ménages. La prise de décision bilatérale des heures de prise de repas garantit la sécurité alimentaire. Lorsque le choix unilatéral du type de repas consommédans les ménages est effectué par les hommes, les ménages sont plus ensécurité alimentaire. Toutefois, les dépenses alimentaires effectuées par l'homme sont plus axées sur les aliments de base, et ne participent pas à la diversité alimentaire. Par contre, les femmes dépensent plus dans les aliments de complément tels que les produits maraîchers, laitiers, etc. Ainsi, les femmes assurent une meilleure diversification alimentaire dans leurs ménages. Les recherches futures pourront se pencher sur le fonctionnement et le partage des dépenses alimentaires entre l'homme et la femme dans les ménages.

\section{Conflits d'intérêts}

Les auteurs ne déclarent aucun conflit d'intérêts en relation avec cet article.

\section{Contributions des auteurs}

Janvier Egah: recherche bibliographique, collecte et traitement des données et rédaction du manuscrit. Ibrahim El Ghazi: rédaction et relecture du manuscrit. Mohamed Nasser Baco: relecture du manuscrit. Marie-Paule Kestemont: supervision de l'étude et validation du manuscrit.

\section{Remerciement}

Nous remercions 1'Académie de Recherche et d'Enseignement Supérieu (ARES) du Gouvernement belge pour le financement de notre séjour posdoctoral à l'Université Catholique de Louvain en Belgique.

\section{References:}

1. Aboubacar, Z. (2017). Participation Politique Des Femmes Au Niger: Analyse De La Contribution Du Genre Féminin Dans Le Développement Politique. European Scientific Journal, 13 (16): 99112. 
2. B2A. (2016). Les Pôles de développement agricole: Approche d'opérationnalisation, 20p.

3. Barou, J., \& Verhoeven, M. (1997). Alimentation et rôles familiaux: la cuisine familiale des immigrés africains. Ethnologie française, 27 (1): 96-102.

4. Baye M., L, (2018). Perspectives Économiques en Afrique 2018. Note Pays.

5. Dury, S., Vall E., \& Imbernon, J. (2017). Production agricole et sécurité alimentaire en Afrique de l'Ouest. Cahiers Agriculture, 26(6): $1-4$.

6. FAO \& Commission de la CEDEAO. (2018). Profil National Genre des Secteurs de l'Agriculture et du Développement Rural - Bénin. Série des Evaluations Genre des Pays. Cotonou. 148 p.

7. FAO, FIDA, OMS, PAM \& UNICEF. (2020). L'État de la sécurité alimentaire et de la nutrition dans le monde 2020. Transformer les systèmes alimentaires pour une alimentation saine et abordable. Rome, FAO, 352p. https://doi.org/10.4060/ca9692fr

8. FAO. (2016). Methods for estimating comparable rates of food insecurity experienced by adults throughout the world. Rome, FAO. $60 \mathrm{p}$.

9. Farzaneh, H., Gargari, B. P., Jafarabadi, M. A., Farzaneh, A., \& Arzhang, P. (2017). Household food insecurity and its related socioeconomic and nutritional factors among northwest Iranian highschool students. Rawal Medical Journal, 42(2), 239-245.

10. Felker-Kantor, E., \& Wood, C. H. (2012). Female-headed households and food insecurity in Brazil. Food Security, 4(4), 607-617.

11. INSAE. (2016). Quatrième Recensement Général de la Population et de l'Habitat (RGPH 4).

12. INSAE \& PAM. (2017). Analyse Globale de la Vulnérabilité, de la Sécurité Alimentaire et Nutritionnelle (AGVSA), 173p.

13. Le Pape, M. C., \& Plessz, M. (2017). C'est l'heure du petit-déjeuner? Rythme des repas, incorporation et classe sociale. L'Année sociologique, 67(1), 73-106.

14. Le Pape, M. C., \& Plessz, M. (2019). Faire petit-déjeuner les enfants. Un enjeu de respectabilité parentale dans les milieux populaires. Revue des politiques sociales et familiales, (129-130), 91-99.

15. Lourme-Ruiz, A., Dury, S., \& Martin-Prével, Y. (2016). Consommet-on ce que l'on sème ? Relations entre diversité de la production, revenu agricole et diversité alimentaire au Burkina Faso. Cahiers Agricultures, 25 (6), 65001.

16. Mohamed-Katerere, J. C., \& Smith, M. (2013). Le rôle des écosystèmes dans la sécurité alimentaire. unasylva, 64(2), 241. 
17. Oussou, K. A., Karamoko, Y., \& Zouh Bi. Z. F. (2017). Rôle des éleveurs de caprins dans l'émergence des maladies caprines (Capra hircus, schaller, 1977) dans la localité de Beoumi (Côte d'ivoire). Journal of Applied Biosciences, 114: 11317-11323.

18. Pauzé, E. (2015). La qualité de l'alimentation et l'accès alimentaire des ménages vulnérables habitant dans une zone d'intervention d'agriculture de santé publique en Haïti, $180 \mathrm{p}$.

19. Verschuur, C. (2011). Changements agraires et des rapports de genre. In Du grain à moudre. Genre, développement rural et alimentation. (Dir.) C. Verschuur. 15-28. Actes des colloques genre et développement. Berne : DDC-Commission nationale suisse pour l'UNESCO; Genève : IHEID.

20. Weiant, P., \& Aswani, S. (2006). Premiers effets de la création d'une zone marine protégée en milieu communautaire sur la sécurité alimentaire des ménages concernés. Ressources marines et traditions, Bulletin de la CPS, 19: 16-31. 